\title{
The Relation Between Vaginal Bleeding During Pregnancy and Preterm Birth in Patients Admitted to Martyrs Hospital in Persian Gulf
}

\author{
Elham Rahmani ${ }^{1}$, Shahnaz Ahmadi ${ }^{1,2^{*}}$, Niloofar Motamed $^{3}$, Najmeh Nasiri Khormoji ${ }^{4}$
}

\begin{abstract}
Objectives: Bleeding during pregnancy, especially in the first trimester, is among the most stressful cases for both patients and physicians. Sometimes bleeding during the first trimester is a predictor of adverse outcomes of pregnancy .The purpose of this study is to document the relationship between vaginal bleeding and preterm birth in patients admitted to the University Hospital in Bushehr.

Materials and Methods: The study was conducted on 1045 pregnant women. All relevant information (including age, parity, gestational age, education level, occupation, history of abortion, preterm delivery, smoking, drugs related to the mother's disease, bleeding, rupture of membrane, mother's blood pressure and vital signs, and gestational age at delivery) was fully recorded in the questionnaire. Then data was analysed by $t$ test, chi-square, and logistic regression in SPSS version 16 software.

Results: There was significant difference between two groups of women with and without pregnancy bleeding $(P<0.05)$ in terms of risk of preterm birth. The vaginal bleeding of first trimester had no significant relation with second trimester in terms of occurrence of preterm birth $(P=0.115)$. The vaginal bleeding had no relation with preterm rupture of membranes $(P=0.0001)$.

Conclusion: The emergence of preterm birth increases in patients with bleeding during the first and second trimesters.

Keywords: Vaginal bleeding, Preterm birth, Preterm premature rupture of the membranes
\end{abstract}

\section{Introduction}

The normal human gestation period is 37 to 42 weeks of pregnancy. Preterm birth refers to birth before 37 weeks of pregnancy. Its prevalence has been calculated to be $15 \%$ at present $(1,2)$ and thus it continues to be a major health problem. This can be attributed to a wide range of causes including maternal, foetal, and placental. About one-third of preterm births occur due to complications in mother or foetus and health risks such as high blood pressure, bleeding or intrauterine growth restriction, cervical failure, and uterine problems. Two-thirds of preterm births occur spontaneously (1,3-4). The most common cause of mortality in developed countries is preterm birth. More importantly, permanent disabilities are seen in infants surviving death. Although care before and after delivery has been effective in improving infant survival, preterm birth still counts for $70 \%$ of infant mortality and $75 \%$ of infant disabilities $(1,2,5)$. It is the major cause of disability for infants regardless of forms of congenital abnormalities $(1,6)$. The problem of preterm birth is the most important reason for admission to the hospital during pregnancy $(1,7)$. It also creates abundant economic problems for families $(1,8)$, and, in spite of all efforts for prevention, its infection rate continues to be on the rise (1). Successful pregnancy depends on the integration of complicated genetic, hormonal, immunological and cellular factors. All these factors should fully cooperate with each other during pregnancy so that fertilization, implantation, and development of the embryo eventually reach fulfilment $(1,9)$. Bleeding during pregnancy, especially in the first trimester, is among the most stressful cases for both patients and physicians. Spotting or bleeding during pregnancy is prevalent especially in the first trimester, often with no reason at all.

It can be due to the implantation of embryos, abortion, ectopic pregnancy, hydatid mole form, changes in the cervix, infection, focal lesions such as polyps and fibroids, and bleeding. Severe bleeding can endanger pregnancy and so this topic needs further consideration. In half of the cases, vaginal bleeding in the first trimester of pregnancy results in spontaneous abortion (10), while women who remain pregnant experience the risk of consequent complications during pregnancy. In general, bleeding during the first trimester is a predictor of adverse outcomes of pregnancy (11) and increases the risk of foetal or neonatal death at least four times (12). Such bleeding caused by

Received 18 April 2016, Accepted 15 August 2016, Available online 15 September 2016

${ }^{1}$ Associate Professor, Fellowship of Infertility, Department of Gynecologic Obstetric, Bushehr University of Medical Sciences, Bushehr, Iran. ${ }^{2}$ Associate Professor, Fellowship of infertility. Department of Gynecologic Obstetric, Iran University of Medical Sciences, Tehran, Iran. ${ }^{3}$ Associate Professor of Community, Department of Community Medicine, Bushehr University of Medical Sciences, Bushehr, Iran. ${ }^{4}$ Medical Student, Bushehr University of Medical Sciences, Bushehr, Iran.

*Corresponding Author: Shahnaz Ahmadi, Tel: +989173717981; Email: Ahmadishahnaz2005@yahoo.com 
emerging disorders accompanies complications such as bleeding during the second and third trimesters, placenta abruption, and foetal growth problems in the womb, preterm rupture of membranes, preterm birth, and low birth weight (13-15). Any bleeding during pregnancy is abnormal, which could be categorized on the basis of the severity of bleeding into spotting, less than menstruation bleeding, as much as menstrual bleeding, and more than menstrual bleeding (1). In case of vaginal bleeding in early pregnancy, the rate of adverse outcomes in later stages of pregnancy increases. According to several studies, bleeding during six to 12 weeks (minor and sever bleeding) has relation with the advent of labour preterm, placental abruption, and spontaneous abortion before 24 weeks (1). The present paper aims to study the relationship between vaginal bleeding and preterm birth in patients admitted to the University Hospital in Bushehr.

\section{Material and Methods}

In a retrospective cohort study, the statistical population included the women admitted to Maternity Clinic of Martyrs hospital in Bushehr from February 2013 to January 2014. The study was conducted on 1045 pregnant women. The inclusion criterion of this study was that all admitted patients should be older than 18 and younger than 40 years with singleton pregnancy and normal blood pressure. Anybody with any kind of infection, diabetes, preeclampsia, placenta previa and placental abruption, past history of preterm delivery, uterine anomalies, and cigarette consumption was excluded. The relevant information was gathered with the help of a questionnaire, a set of interviews, and full investigation of records of these patients. The patients participated in the study after giving written consent. All relevant information was fully recorded in the questionnaire. The admission and delivery time for each patient was registered in the maternity hospital. At first, after thorough checking of medical history and performing ultrasounds along with other necessary laboratory tests, the patients were given essential tips and told about warning signs, including signs of bleeding, pain, and infection. According to routine, the time schedule of next prenatal visits was organized on the basis of instructions till the 36 th week and then once a week. Further monitoring of the patients was performed in hospital clinic and maternity hospitals at admission time. The information was registered in the personal questionnaire and the remaining data was extracted from medical records of the patients. In this respect, necessary variables, including age, parity, gestational age based on ultrasounds and the date of the last menstrual period, education level, occupation, history of abortion, preterm delivery, smoking, drugs related to the mother's disease, bleeding, bleeding episodes, intensity bleeding, premature rupture of membranes (PROM), mother's blood pressure and vital signs, and gestational age at delivery, are determined.

\section{Statistical Analysis}

Data was expressed through mean, standard deviation
(SD), and absolute and relative frequency, and was analysed by $t$ test, chi-square, and logistic regression in SPSS version 16 software (SPSS Inc., Chicago, IL, USA). Confidence interval of 95 and $P$ values of less than 0.05 were considered to indicate statistical significance.

\section{Results}

From the 1045 women considered for this study, 35 were excluded because their deliveries were conducted in another hospital and there was no possibility to communicate with them. Among the remaining women, five had hardworking conditions. As many as $67.9 \%$ of the participants had less than three children, and 184 cases of abortion had been recorded. The frequency distribution of demographic and fertility collective variables among the participants was showed in Table 1. Among 1010 women, 260 were involved in preterm birth and 306 in preterm rapture of membranes, while 201 women had vaginal bleeding. The highest reported bleeding was related to spotting (Table 2). According to Tables 3 and 4, hardworking condition $(P=0.17, \mathrm{CI}$ : $1.3-105.18)$, vaginal bleeding reports $(P=0.0001, \mathrm{CI}: 5.75-11.34)$, severity of vaginal bleeding $(P=0.0001)$, and preterm rapture $(P=0.0001, \mathrm{CI}$ : 2.05-3.71) were significantly related with preterm birth. There was no significant relation between age, gravidity, abortion report, pregnancy age, and preterm birth. But, in general, there is significant relation $(P=0.0001, \mathrm{CI}$ : 7.39-148.39) between vaginal bleeding pregnancy period and preterm rapture of membranes (Table 5). By equalization of patients in terms of age, gravidity, hardworking

Table 1. Frequency Distribution of Demographic and Fertility Collective Variables in Women Participating the Study in Bushehr (2013-2014)

\begin{tabular}{lccc}
\hline Variable & & Number & Percent \\
\hline \multirow{2}{*}{ Hard work } & Yes & 5 & 0.5 \\
History from & No & 1005 & 99.5 \\
abortion & Yes & 184 & 18.2 \\
Gravidity & No & 826 & 81.8 \\
& $<3$ & 686 & 67.9 \\
\hline
\end{tabular}

Table 2. Frequency Distribution of Preterm Birth, Preterm Rapture of Membranes, Vaginal Bleeding Report, Sever Vaginal Bleeding, Pregnancy Age of Vaginal Bleeding and Age of Patients

\begin{tabular}{|c|c|c|c|}
\hline Variable & & Number & Percent \\
\hline \multirow{2}{*}{ Preterm Labor } & Yes & 260 & 25.7 \\
\hline & No & 750 & 74.3 \\
\hline \multirow{2}{*}{$\begin{array}{l}\text { Premature rupture } \\
\text { of membrane }\end{array}$} & Yes & 306 & 30.1 \\
\hline & No & 704 & 69.9 \\
\hline \multirow{2}{*}{ History of bleeding } & Yes & 201 & 20.1 \\
\hline & No & 809 & 79.9 \\
\hline \multirow{3}{*}{ Amount of bleeding } & Spotting & 168 & 16.6 \\
\hline & Menstruation & 29 & 2.9 \\
\hline & More of menstruation & 5 & 0.5 \\
\hline \multirow{3}{*}{$\begin{array}{l}\text { Gestational age of } \\
\text { bleeding }\end{array}$} & NO & 809 & 80.1 \\
\hline & $14 \mathrm{wk}$ & 177 & 17.5 \\
\hline & $14-27 w k$ & 24 & 2.4 \\
\hline \multirow[t]{2}{*}{ Age of patient } & $<35 y$ & 880 & 87.1 \\
\hline & $\geq 35 y$ & 130 & 12.9 \\
\hline
\end{tabular}


Table 3. The Relation Between the Variables of Hard Working Condition, Vaginal Bleeding, Abortion Report, and Preterm Birth in Women Under Study

\begin{tabular}{|c|c|c|c|c|c|c|c|}
\hline \multirow{2}{*}{ Variable } & & \multicolumn{2}{|c|}{ Preterm Labor } & \multirow{2}{*}{ P Value } & \multirow{2}{*}{$\begin{array}{l}\text { Odds } \\
\text { Ratio }\end{array}$} & \multicolumn{2}{|c|}{$95 \% \mathrm{Cl}$} \\
\hline & & Yes & No & & & Lower & Upper \\
\hline \multirow{2}{*}{ Hard work } & Yes & $4(80 \%)$ & $1(20 \%)$ & $0.017^{a}$ & \multirow{2}{*}{11.7} & \multirow{2}{*}{1.3} & \multirow{2}{*}{105.18} \\
\hline & No & $256(25.5 \%)$ & 749 (74.5\%) & & & & \\
\hline \multirow{2}{*}{ Abortion } & Yes & $57(31 \%)$ & 127 (69\%) & $0.077^{a}$ & \multirow{2}{*}{1.37} & \multirow{2}{*}{0.97} & \multirow{2}{*}{1.95} \\
\hline & No & $203(75.4 \%)$ & $623(24.6 \%)$ & & & & \\
\hline \multirow{2}{*}{ Gravid } & $<3$ & $188(27.4 \%)$ & $498(72.6 \%)$ & $0.09^{a}$ & \multirow{2}{*}{1.321} & \multirow{2}{*}{0.968} & \multirow{2}{*}{1.803} \\
\hline & $\geq 3$ & $72(22.2 \%)$ & $252(77.8 \%)$ & & & & \\
\hline \multirow{2}{*}{ Gestational bleeding } & Yes & 125 (61.9\%) & $76(38.1 \%)$ & $0.0001^{\mathrm{a}}$ & \multirow{2}{*}{8.08} & \multirow{2}{*}{5.75} & \multirow{2}{*}{11.34} \\
\hline & No & $135(16.7 \%)$ & $673(83.3 \%)$ & & & & \\
\hline
\end{tabular}

a Fisher exact test.

Table 4. The Relation Between Vaginal Bleeding, and Severity of Bleeding, Rapture of Membranes, Abortion Report, Pregnancy Age and Preterm Birth in Women Under Study

\begin{tabular}{|c|c|c|c|c|c|c|c|}
\hline \multirow{2}{*}{ Variable } & & \multicolumn{2}{|c|}{ Preterm Labor } & \multirow{2}{*}{$P$ Value } & \multirow{2}{*}{ Odds Ratio } & \multicolumn{2}{|c|}{$95 \% \mathrm{Cl}$} \\
\hline & & Yes & No & & & Lower & Upper \\
\hline \multirow{2}{*}{$\begin{array}{l}\text { Premature rupture of } \\
\text { membrane }\end{array}$} & Yes & $122(40.1 \%)$ & 182 (59.9\%) & \multirow{2}{*}{$0.0001^{a}$} & \multirow{2}{*}{2.76} & \multirow{2}{*}{2.05} & \multirow{2}{*}{3.71} \\
\hline & No & $138(19.5 \%)$ & $568(80.5 \%)$ & & & & \\
\hline \multirow{2}{*}{ History of bleeding } & Yes & $125(62 \%)$ & $76(38 . \%)$ & \multirow{2}{*}{$0.0001^{a}$} & \multirow{2}{*}{8.08} & \multirow{2}{*}{5.75} & \multirow{2}{*}{11.34} \\
\hline & No & $135(16.7 \%)$ & $672(83.3 \%)$ & & & & \\
\hline \multirow{4}{*}{ Amount of bleeding } & No & $135(16.7 \%)$ & $674(83.3 \%)$ & \multirow{4}{*}{$0.0001^{b}$} & \multirow{4}{*}{-} & \multirow{4}{*}{ - } & \multirow{4}{*}{-} \\
\hline & Spotting & $104(61.9 \%)$ & $64(38.1 \%)$ & & & & \\
\hline & Menstruation & $18(62.1 \%)$ & $11(37.9 \%)$ & & & & \\
\hline & More of menstruation & $3(75 \%)$ & $1(25 \%)$ & & & & \\
\hline \multirow{2}{*}{ Age } & $<35 y$ & $225(25.6 \%)$ & $655(74.4 \%)$ & \multirow{2}{*}{$0.748^{\mathrm{a}}$} & \multirow{2}{*}{1.07} & \multirow{2}{*}{0.7} & \multirow{2}{*}{1.62} \\
\hline & $\geq 35 \mathrm{y}$ & $35(26.9 \%)$ & $95(73.1 \%)$ & & & & \\
\hline \multirow{2}{*}{ Gestational age of bleeding } & $14 \mathrm{wk}$ & $114(64.4 \%)$ & $63(35.6 \%)$ & \multirow{2}{*}{$0.115^{\mathrm{a}}$} & \multirow{2}{*}{2.139} & \multirow{2}{*}{0.905} & \multirow{2}{*}{5.053} \\
\hline & $14-27 w k$ & $11(45.8 \%)$ & $13(54.2 \%)$ & & & & \\
\hline
\end{tabular}

${ }^{\mathrm{a}}$ Fisher exact test; ${ }^{\mathrm{b}}$ Chi-square test.

Table 5. Relation Between Vaginal Bleeding and Preterm Rapture of Membrane in This Study

\begin{tabular}{|c|c|c|c|c|c|c|c|}
\hline \multirow{2}{*}{ Variable } & & \multicolumn{2}{|c|}{ Premature of Membrane } & \multirow{2}{*}{$P$ Value } & \multirow{2}{*}{ Odds Ratio } & \multicolumn{2}{|c|}{$95 \% \mathrm{Cl}$} \\
\hline & & Yes & No & & & Lower & Upper \\
\hline Gestational bleeding & Yes & $145(72.1 \%)$ & $56(27.9 \%)$ & $0.0001^{* *}$ & 10.42 & 7.39 & 148.39 \\
\hline
\end{tabular}

Table 6. The Adjusted Odds Ratio and $\mathrm{Cl}$ of $95 \%$ in Terms of Age, Gravidity, Hard Working Condition, and Abortion Report for The Impact of Independent Variables on Dependent Variable (Preterm Birth)

\begin{tabular}{|c|c|c|c|c|c|c|}
\hline \multirow{2}{*}{ Variable } & & \multirow{2}{*}{ B } & \multirow{2}{*}{ P value } & \multirow{2}{*}{ Odds Ratio } & \multicolumn{2}{|c|}{$95 \% \mathrm{Cl}$} \\
\hline & & & & & Lower & Upper \\
\hline Premature of rupture membrane & & 1.18 & 0.0001 & 3.28 & 2.34 & 4.58 \\
\hline \multirow{2}{*}{ Gestational age of bleeding } & $14 \mathrm{wk}$ & 2.31 & 0.0001 & 10.1 & 6.87 & 14.82 \\
\hline & $14-27 w k$ & 1.5 & 0.001 & 4.51 & 1.9 & 10.73 \\
\hline
\end{tabular}

condition, medicine consumption, and abortion report, the adjusted odds ratio of membrane rapture and preterm birth is 3.28 , and the adjusted odds ratio is 10.1 for the first trimester and 4.51 for the second trimester (Table 6).

\section{Discussion}

The present study shows that hardworking conditions have direct relation with preterm birth. The record of abortion has no impact on preterm birth, and the reason may be the omission of reasons, such as uterine abnormalities, that are effective in preterm birth. After the omission of these trouble-making factors, the relation between bleeding during the first and second trimesters and preterm birth was studied. The result showed that the probability of occurrence of preterm birth in the vaginal bleeding group increased 8.94 times and its real value with 95\% CI is at least 6.26 to 12.77 . The probability of preterm birth in the first trimester (CI: 6.87-14.82, OR: 10.1) is more than that in second trimester (OR: 4.51, CI: 1.9-10.73). Sharami et al (16) indicated that vaginal bleeding has been associated with an increased 3-fold risk of preterm birth. They also showed that the feature of bleeding, including time, frequency, and severity, has significant relation with preterm birth, which is consistent with our study. The study by Amirkhani et al (17) also verifies this relation. The study conducted by Arafa et al in 1503 singleton preg- 
nancies reported $10.6 \%$ vaginal bleeding during pregnancy, of which $63.5 \%$ was during the first trimester and $36.5 \%$ during the second trimester. Here vaginal bleeding, abortion, and preterm labour were more common among women $\geq 35$ years. The results were less than desirable among women who reported more bleeding than those who never had bleeding, and, therefore, the risk of such significant results show increases in bleeding in the second trimester. For women with first trimester bleeding or second trimester bleeding, early foetus loss is significantly reduced by increasing the interval between pregnancies (18). Leung et al showed that unexplained bleeding before birth (before 34 weeks of gestation) is associated with high risks of preterm delivery (29.3\%) (19).

In our study, the spotting and bleeding increased the risk of preterm birth, which is consistent with the study by Weiss et al (20). The study by Edwards et al (21) indicated that bleeding in the form of spotting is not consistent with preterm birth and only severe bleeding could lead to an increment in preterm birth. Vaginal bleeding could actually move towards thrombin and proteolytic cascades, which could damage foetal membranes and lead to preterm rupture of such membranes $(22,23)$. Thrombin stimulates uterine contractions. Besides the direct impact, bleeding could also be a manifestation of subclinical infection and inflammation of the uterus, which could lead to preterm birth (23). According to this study conducted on patients, vaginal bleeding in the first and second trimesters was associated with preterm rupture of membranes. However, the study conducted by Hossain et al (24), which considered 2678 cases of birth, reported no preterm rupture of membranes. But here preterm birth was associated with first trimester bleeding. However, the study by Yang et al (25) on 56 women with vaginal bleeding in the first and second trimesters associates vaginal bleeding with the risk of preterm birth regardless of the amount and time of bleeding reported two times more and the risk of preterm rupture of membranes in women with record of bleeding in the first trimester reported to be more than control group. Bleeding with demolition of chorioamniotic space and chronic inflammation probably accelerates preterm birth (26). The retroperitoneal hematoma pair could also lead to uterine contractions, which is consistent with our study. Coleman et al (27) believed that if women exposed to the risk of preterm birth could be identified, then they would have better prenatal prognosis.

\section{Conclusion}

The risk of preterm birth in two target and test groups showed significant differences, as found by the present study. The emergence of preterm birth increases in patients with bleeding during the first and second trimesters. With respect to the related impacts during the first and second trimesters on the emergence of preterm birth and preterm rapture of membranes, it seems that a prescription of progesterone suppository with fewer side effects, instead of beta agonists, would prevent the consequent complications.
Ethical Issues

Approval for this study was received from the ethics committee of Bushehr University.

\section{Confilict of Interests}

None.

\section{Financial Support}

None.

\section{Acknowledgments}

The authors are grateful to the Bushehr University of Medical Sciences and vice-chancellor of the research \& technology affairs for approval of the proposal. The relevant data was collected from Miss Nasiri's thesis.

\section{References}

1. Cunningham F, Leveno KJ, Bloom SL, Hauth JC, Rouse DJ, Spong CY. Abortion. In: Williams Obstetrics. New York: McGraw Hill; 2010.

2. Danforth DN, Gibbs RS. Danforth's Obstetrics and Gynecology. Lippincott Williams \& Wilkins; 2008.

3. Firouzabadi RD, Rahmani E, Rahsepar M, Firouzabadi MM. Value of follicular fluid vitamin D in predicting the pregnancy rate in an IVF program. Arch gynecol obstet. 2014;289:201-6. doi: 10.1007/ s00404-013-2959-9.

4. Tajbakhsh S, Esfahani MN, Emaneini M, Motamed N, Rahmani E, Gharibi S. Identification of Streptococcus agalactiae by fluorescent in situ hybridization compared to culturing and the determination of prevalence of Streptococcus agalactiae colonization among pregnant women in Bushehr, Iran. BMC Infect Dis. 2013;13:420. doi: 10.1186/1471-2334-13-420.

5. Eftekhar M, Rahsepar M, Rahmani E. Effect of progesterone supplementation on natural frozenthawed embryo transfer cycles: a randomized controlled trial. Int J Fertil Steril. 2013;7:13-20.

6. Davar R, Rahsepar M, Rahmani E. A comparative study of luteal estradiol pre-treatment in $\mathrm{GnRH}$ antagonist protocols and in micro dose flare protocols for poor-responding patients. Arch Gynecol Obstet. 2013;287:149-53. doi: 10.1007/s00404-012-2522-0.

7. Eftekhar M, Rahmani E, Eftekhar T. Effect of adding human chorionic gonadotropin to the endometrial preparation protocol in frozen embryo transfer cycles. Int J Fertil Steril. 2012;6(3):175-8.

8. Eftekhar M, Rahmani E, Pourmasumi S. Evaluation of clinical factors influencing pregnancy rate in frozen embryo transfer. Iran J Reprod Med. 2014;12:513.

9. Ahmadi S, Rahmani E, Motamed N, Ghorbanpoor M, Maneshi H. Bishop score predictive value in success of induced labor process among full term pregnant women referred to Persian Gulf Martyrs' Hospital in Bushehr in 2013. Iran South Med J. 2016;19:620-8.

10. De Sutter P, Bontinck J, Schutysers V, Van der Elst J, Gerris J, Dhont M. First-trimester bleeding and pregnancy outcome in singletons after assisted 
reproduction. Hum Reprod. 2006;21:1907-11. doi: 10.1093/humrep/del054.

11. Paspulati RM, Bhatt S, Nour S. Sonographic evaluation of first-trimester bleeding. Radiol Clin North Am. 2004;42:297-314. doi: 10.1016/j.rcl.2004.01.005.

12. Saraswat L, Bhattacharya S, Maheshwari A. Maternal and perinatal outcome in women with threatened miscarriage in the first trimester: a systematic review. BJOG. 2010;117:245-57. doi: 10.1111/j.14710528.2009.02427.

13. Kim YJ, Lee BE, Park HS, Kang JG, Kim JO, Ha EH. Risk factors for preterm birth in Korea. Gynecol Obstet Invest. 2005;60:206-12. doi: doi:10.1159/000087207.

14. Madan I, Romero R, Kusanovic JP, et al. The frequency and clinical significance of intra-amniotic infection and/or inflammation in women with placenta previa and vaginal bleeding: an unexpected observation. J Perinat Med. 2010;38:275-9. doi: 10.1515/jpm.2010.001.

15. Rahmani E, Ahmadi S, Motamed N, Yazdani N. Study of association between ovarian volume with the number of antral follicles and third day of menstruation $\mathrm{FSH}$ in infertile patients referred to Omid Persian gulf infertility Clinic. Iran South Med J. 2016;19:608-19.

16. Sharami SH, Darkhaneh RF, Zahiri Z, et al. The relationship between vaginal bleeding in the first and second trimester of pregnancy and preterm labor. Iran J Reprod Med. 2013;11:385.

17. Amirkhani Z, Akhlaghdoust M, Abedian M, et al. Maternal and Perinatal Outcomes in Pregnant Women with First Trimester Vaginal Bleeding. J Family Reprod Health. 2013;7:57.

18. Arafa M, Abdel-Fataah M, Zeid H, El-Khouly A. Outcomes of pregnancies complicated by early vaginal bleeding. East Mediterr Health J. 2000;6:45764.

19. Leung TY, Chan LW, Tam WH, Leung TN, Lau
TK. Risk and prediction of preterm delivery in pregnancies complicated by antepartum hemorrhage of unknown origin before 34 weeks. Obstet Gynecol Invest. 2001;52:227-31. doi: 10.1159/000052980.

20. Weiss JL, Malone FD, Vidaver J, et al. Threatened abortion: a risk factor for poor pregnancy outcome, a population-based screening study. Am J Obstet Gynecol. 2004;190:745-50. doi: 10.1016/j. ajog.2003.09.023.

21. Edwards DV, Baird D, Hasan R, Savitz D, Hartmann K. First-trimester bleeding characteristics associate with increased risk of preterm birth: data from a prospective pregnancy cohort. Hum Reprod. 2012; 27:54-60. doi: 10.1093/humrep/der354.

22. Elovitz MA, Baron J, Phillippe M. The role of thrombin in preterm parturition. Am J Obstet Gynecol. 2001;185:1059-63. doi: 10.1067/mob.2001.117638.

23. Lockwood CJ, Kuczynski E. Markers of risk for preterm delivery. J Perinat Med. 1999;27:5-20. doi: 10.1515/JPM.1999.001.

24. Hossain R, Harris T, Lohsoonthorn V, Williams MA. Risk of preterm delivery in relation to vaginal bleeding in early pregnancy. Eur J Obstet Gynecol Reprod Biol. 2007;135:158-63. doi: 10.1016/j.ejogrb.2006.12.003.

25. Yang J, Hartmann KE, Savitz DA, et al. Vaginal bleeding during pregnancy and preterm birth. Am J Epidemiol. 2004;160:118-25. doi: 10.1093/aje/ kwh180.

26. Arslan N, Kibrisli E, Findik FM, Yilmaz A, Aslanhan H. Vaginal birth after repeated cesrean section: a case report. International Journal of Women's Health and Reproduction Sciences. 2016;4(3):146-8. doi: 10.15296/ijwhr.2016.33.

27. Coleman MA, McCowan LM, Pattison NS, Mitchell M. Fetal fibronectin detection in preterm labor: evaluation of a prototype bedside dipstick technique and cervical assessment. Am J Obs Gyn. 1998;179: 1553-8. doi: 10.1016/S0002-9378(98)70023-7.

Copyright ( 2016 The Author(s); This is an open-access article distributed under the terms of the Creative Commons Attribution License (http://creativecommons.org/licenses/by/4.0), which permits unrestricted use, distribution, and reproduction in any medium, provided the original work is properly cited. 